\title{
PATHOLOGICAL AND SURGICAL
}

\section{OBSERVATIONS}

\author{
RELATING TO
}

\section{INJURIES OF THE BRAIN;}

\author{
By B. C. BRODIE, F.R.S.
}

AND SURGEON TO ST. GEORGE'S HOSPITAL.

\section{PART I.}

Read 26th February, 11th and 25th March, 1828.

\section{SECT. 1.}

IT is my intention in the following pages to lay before the Society some observations relating to injuries of the brain, and the treatment which these injuries require. If any apology be necessary for this undertaking, I may remark, that I have been led to it by the great importance of the subject, and also by this consideration, that, although much information may lie, as it were, scattered throughout the mass of surgical literature, no practical writer, as far as I know, has attempted to make such a collection and arrangement of facts as will enable the surgical student to take a distinct and connected view of all the parts of this curious and interesting inquiry. The present paper contains only a part of the observ-

vol. XIV. 
ations which I have to offer, namely, those which relate to the first or immediate consequences of the injury. Should these be favourably received, I shall venture, on a future oecasion, to communicate to the Society a second series of observations relating to those more remote consequences which are connected with inflammation of the brain and its membranes, or which arise after inflammation has subsided.

SEcT. 2.-Immediate Effects of Injuries of the Head as indicated by Dissection.

In treating of injuries of the brait, of course I mean to include the consideration, not only of those by which the brain is affected in a direct, but also of those by which it is affected in an indirect, manner. Wounds and contusions of the external parts of the head demand our attention, inasmuch as they may be, and not unfrequently are, followed by disease of the more important parts contained within. Among the effects produced we are to distinguish those which are the immediate result of the injury, and those which are to be attributed to inflammation and its consequences. In the former we are still further to distinguish the actual derangement or destruction of the natural organization, such as it is disclosed by dissection, and the symptoms produced during the life of the patient by the disturbance of the functions of the injured organ; attempting at the 
same time to view these two orders of facts in connection with each other, as the method by which, on this as well as on other occasions, we may be the best enabled to found the practical art of surgery on the basis of a scientific pathology.

The appearances which are observable on dissection in a person, who dies soon after an injury of the head, are very various, and may be variously complicated; but they admit of being classed under the following heads :

1. There may be simple contusion of the scalp with extravasation of blood between it and the tendon of the occipito-frontalis muscle, or between the latter and the pericranium, or between the pericranium and the bone; concerning which it is scarcely necessary to repeat the observation of $\mathrm{Mr}$. Pott as to the close resemblance of the impression which is given to the fingers by the margin of the mass of extravasated blood, and that of depressed bone.

2. The scalp may be lacerated so as to expose the surface of the pericranium, or the pericranium itself. may be torn off with it so as to expose the surface of the bone. Of these injuries, however slight may be the apparent difference between them, the latter is, as I shall shew hereafter, likely to produce much more serious consequences than the former. 
3. If a blow be inflicted on the head of the dead subject, the small vessels which connect the dura mater to the inside of the bone, at the part where the blow is inflicted, become ruptured; and in consequence the dura mater is separated from the bone to a greater or less extent. This, which happens in the dead body, may happen in the living body also, and is not an unfrequent consequence of an injury of the head. The separation of the dura mater is sometimes very extensive. A boy twelve years of age, fell from a height of fifty feet, and struck his forehead against the ground. He was admitted into St. George's Hospital in a state of stupor, in which he lay for three days, when he died. On dissection, besides a large extravasation of blood on the inferior surface of the brain, the dura mater was found to have lost its adhesion to the bone everywhere, except in the basis of the cranium, and the external surface of that membrane had a brown and sloughy appearance.

4. The cranium may be fractured in all varieties of ways, from the most simple fissure to the most complicated fracture accompanied with depres-. sion and extending in a number of directions. A fracture in most instances takes place in the upper part of the cranium. Fractures of the basis are always the consequence of very severe contusion, and recoveries from these accidents are comparatively rare, not because a fracture of the basis is in 
itself more dangerous than a fracture elsewhere, but because it is almost invariably complicated with extensive injury of other and more important parts.

A fracture generally occurs in that part of the cranium on which the blow has been inflicted. But we find that in cases of fracture of other bones, the fracture is often situated at some distance from the part which is immediately exposed to the shock of the injury, as when the fibula is broken a little above the outer ankle in consequence of the foot having been twisted outwards, or the ribs are broken in the side in consequence of a blow on the sternum; and some French writers have supposed that fractures of the cranium occur in the same manner, being produced by what they have denominated the contre-coup.

It has been observed to me, however, by $\mathrm{Mr}$. Earle, that he has not known a fracture of this kind to take place except where the blow seems to have operated in such a manner as to impel the occiput forcibly against the atlas, the line of fracture passing through the former bone, where it rests on the latter. My own experience corresponds very nearly with that of Mr. Earle. The only well marked cases of fracture of the cranium, in which the fracture could be attributed to the effects of the contre-coup, which have fallen under my own observation, were similar to those which 
he has mentioned. I do not, however, mean to assert, that such fractures absolutely never occur independent of the re-action of the atlas. Among the cases recorded in the Prize Memoirs of the French Academy of Surgery there are some which shew that the thing does happen*, and Mr. Bell has offered an ingenious and scientific explanation of the mode in which it happens. It is, however, worthy of remark, that the only two cases which Mr. Bell has adduced in illustration of what he has advanced, are those in which the fracture extended across the occiput, in one case passing through, and in the other case passing close to, the foramen magnum of that bone.

In all cases of fracture of the cranium, with depression of bone, it is of importance to observe that the division of the inner does not correspond to that of the outer table of the skull, the former being always broken to a greater extent than the latter. In consequence of this the actual depres-

* For example, M. Saucerote quotes from Joan. Bonhius de Renunt. Vulnerum the following history. A man died after having received a blow above the right eyebrow. On dissection it was ascertained that there was no fracture in the part which had been struck, but in the right orbit there was a fracture half an inch in length, extending towards the Sella Turcica of the sphenoid bone. But with respect to many other cases which are mentioned in the same memoir, it may be said that there is no sufficient evidence that the fracture which was attributed to the counter-stroke, did not really arise from a second blow on another part of the head. 
sion is greater than it.would appear to be from the mere inspection of the external fracture.

I have seen a case in which there was a fracture with distinct depression of the inner table, while there was a simple fissure which was scarcely perceptible, and that without the smallest depression, of the outer table. But more remarkable instances of the kind are recorded by authors. M. Saucerote*, in the Prize Memoirs of the French Academy, quotes a case from Tulpius, in which there were extensive fissures of the inner table of the skull, although the outer table remained uninjured; and another from Parey, in which, while the outer table was entire, the inner table was broken into splinters, some of which were actually driven into the substance of the brain. Dr. Hennen also in his Treatise on Military Surgery $t$, gives an account of a case similar to the last, in which the inner table was splintered, and at one part driven more than half an inch into the membranes of the brain, although there was not even a fissure of the outer table. The greater elasticity of the outer table of the skull, and the greater brittleness of the inner table seem to afford the only reasonable solution of these phænomena.

5. In young children we sometimes find the

* Vol. IV. 8vo edition, 1819, p. 322.

+ P. 323, second edition. 
cranium depressed or indented after a blow on the head, and in the course of a few days restored to its natural level without the aid of a surgeon. I suppose that in these cases the earthy part of the bone has given way, while the animal part has remained entire, so that there has not been a complete fracture or actual solution of continuity, and that the pulsations of the brain constantly operating against the inner surface of the bone have been the means of elevating the depression. I have had no opportunity of verifying or contradicting this opinion by dissection, but it corresponds to what we know to happen in cases of injury done to other bones during the period of childhood.

6. The disjunction of the sutures is much more rare than fractures of the cranium. It is evident that this cannot happen except in those who are not much advanced in life, and in whom the sutures are not completely consolidated. Such a case is always to be regarded as one of peculiar danger, not so much because the disjunction of the sutures is in itself likely to lead to bad consequences, but because the force necessary to produce it is so great, that it is also likely to produce extensive and serious injury of other parts.

7. Extravasations of blood within the cranium, in consequence of a blow on the head, occur in various situations: 1st, between the bone and 
dura mater, and here the extravasation may arise from a rupture of the small blood-vessels by which the dura mater is connected to the bone, or from a laceration of the trunk or branches of the middle meningeal artery. There is however never any considerable hæmorrhage from the former source. At least, all the experience which I have had on the subject tends to confirm the opinion advanced long ago by $\mathrm{Mr}$. Abernethy, that blood is never poured out in such quantity as to produce a dangerous pressure on the brain, except where the middle meningeal artery has been lacerated, and from this vessel the hæmorrhage is sometimes very copious. I do not recollect to have seen it lacerated, except in combination with fracture running across the bony canal in which it is lodged; cases are however recorded by authors, in which the artery has been opened into, and bleeding has taken place from it, independently of fracture*. 2dly, there may be extravasations of blood within the dura mater, and here the blood is generally collected between the dura mater and the tunica arachnoides. Sometimes however, but rarely, the blood occupies the ventricles; at other times we find it extravasated in the substance of the brain, or in the cells of the cellular texture by which the tunica arachnoides and pia mater are connected with each other. Large extravasations are sometimes found on the upper surface

* Two such cases are quoted by Mr. Abernethy, one from Mr. Hill, and the other from Mr. Latta. 
of the brain; but more frequently on its basis. In the latter situation, the hæmorrhage is usually the consequence of a rupture of the substance of the brain. As a blow on the abdomen may lacerate the substance of the liver or spleen, and occasion hæmorrhage into the peritonæal cavity, so may a blow on the head cause a rupture of the tender substance of the cerebrum or cerebellum, and hæmorrhage into the cavity of the dura mater. These cases generally afford examples of the contre-coup. The rupture of the brain rarely takes place at the exact spot at which the blow is inflicted; and the great irregularities which exist on the inner surface of the basis of the cranium, sufficiently explain wherefore the inferior is more liable to be ruptured than the superior surface of the brain.

Wounds of the sinuses sometimes bleed profusely where there is a free opening in the bone made by accident or operation, through which the blood can readily escape. But a very slight pressure is adequate to the suppression of this as well as of other venous hæmorrhage ; and I have never known an instance in which there was such a collection of blood as was capable of interfering with the functions of the brain, between the dura mater and the bone, or between the dura mater and the brain, in consequence of a wounded sinus. There is often a considerable effusion of blood from the ear, especially in cases of fracture of the basis of the cranium. This may, as far as I know, sometimes 
arise from other sources; but it seems probable that it must, in most instances, arise from the laceration of the lateral sinus, where it extends downwards behind the petrous process of the temporal bone and the external meatus; and in one instance I ascertained it to have been so by the examination of the body after death. In another case which fell under my observation, there was hæmorrhage from both the ear and the nostrils. The patient, a boy, died shortly after the accident; and it was found on dissection that there was a fracture of the base of the cranium, with laceration of the cavernous sinus, and that the hæmorrhage had taken place from this sinus.

7. There may be all descriptions of wounds of the brain and its membranes, punctured, incised, and lacerated, with or without loss of substance: and with these, the effects of contusion which have been already enumerated, may be variously combined.

\section{SEct. 3.-Concussion of the Brain.}

It is evident that many of those consequences of an injury of the head which are disclosed to us by dissection, are not likely to be marked by any peculiar symptoms in the living person, at least not previous to the access of inflammation. Wounds and lacerations of the brain, 
and compression of the brain, whether it arise from extravasated blood or a depression of bone, may impair or destroy the functions of that organ; but neither simple fissures of the cranium, nor disjunction of the sutures, nor separation of the pericranium or dura mater, are in themselves adequate to produce such effects in the first instance, although they may lay the foundation of serious disease afterwards.

But it has been long since established by the investigations of surgeons, that another cause, besides those which are rendered manifest by dissection, may be concerned in producing the symptoms which immediately follow a contusion of the head. A man receives a blow on the head; he becomes insensible, and continues so for a few minutes or for several hours. He dies, in consequence of this or some other injury; and on examination after death, the brain and its coverings appear to be perfect in all their parts; so that the most accurate anatomist can discover nothing different from the natural appearance of these organs. Opportunities of verifying this observation occur more or less to all those who have had much experience in their profession. In such cases, the patient is said to have been stunned, or to have suffered from concussion of the brain: and it is to one of these three causes, namely, concussion, compression, and wounds of the brain, 
that the symptoms which immediately follow an injury of the head, and which are antecedent to those produced by inflammation, are to be referred.

Opportunities of inspecting the brain, where the patient has laboured under symptoms of concussion, may arise, 1st, where the concussion has so disturbed the functions of that organ as to have been in itself a cause of death (which is, on the whole, a rare occurrence). 2dly, where the concussion of the brain has been complicated with other and still more serious mischief. We learn from such examinations, that the symptoms which are ascribed to concussion do not depend on any such derangement of the organization as admits of being disclosed to us by dissection. The brain appears to retain its natural structure unimpaired. We are not however justified in the conclusion that there is therefore in reality no organic injury. It is difficult to conceive in what other manner concussion of the brain can operate so as to produce the effects which it is known to produce; and if we consider that the ultimate structure of the brain is on so minute a scale that our senses are incapable of detecting it, it is evident that there may be changes and alterations of structure, which our senses are incapable of detecting also. The speedy subsiding of the symptoms. of concussion does not contradict this opinion. A deep incised wound in other parts of the body may, under certain circumstances, be completely 
and firmly united in the space of twenty-four bours; and it is easy to suppose that the effects of a much slighter injury may be repaired in a still shorter space of time.

The disturbance of the functions of the brain, which is the consequence of concussion may exist in various degrees and may be of various duration.

In many instances there is at first complete insensibility to external impressions. The patient lies as if in a state of apoplexy, from which however he recovers in the course of a few minutes. In some instances the recovery is complete; the patient rises and walks away as if nothing unusual had occurred. In other cases this state of total insensibility is followed by one in which the sensibility is impaired, but not destroyed. The patient is not affected by ordinary impressions, but if spoken to in a loud tone of voice, he will shift his position, and answer in a peevish manner. Sometimes he is in a state of imperfect delirium, talking in an incoherent and rambling manner, as if intoxicated. The pupils contract on exposure to light, and are sometimes more contracted than under ordinary circumstances. There is no paralysis. The respiration in the great majority of cases is performed easily and naturally; in a few instances only it is laboured, and approaching to being stertorous. These symptoms 
may wholly subside in the course of a few hours or they may continue for three or four days. In the latter case it frequently occurs that the patient regains his sensibility for a time, and then relapses into his former condition. Where inflammation of the brain follows the injury done by concussion, it may be that the primary effects of the concussion are entirely relieved, so that there is a considerable interval of sense before the inflammation shews itself. But it may be also that there is no such interval, and the symptoms of concussion, in this last case, are gradually and imperceptibly converted into those of inflammation.

Concussion of the brain in almost every instance occasions head-ach; sometimes a slight headach, which is speedily relieved; at other times an intense head-ach, which may remain for some days, a solitary symptom, after all other symptoms are vanished. Sickness and vomiting for the most part are early symptoms, and seldom continue after the patient has recovered from the first shock of the accident. Of course there is no recollection afterwards of what occurred during the period of complete insensibility. The memory however is sometimes affected to a still greater extent; , and the impressions made on the mind by the events immediately antecedent to the injury become obliterated. A groom in the employment of the Persian ambassador, in the summer of 1819 , 
was engaged in cleaning one of the ambassador's horses, when he received a kick from the animal on the head. He did not fall, nor was he actually insensible or stunned; but he entirely forgot in what employment he had been engaged at the time of receiving the blow. Being unable to account for the time which had elapsed, he concluded that he had been asleep: said so to his fellow-servanț, observing at the same time that "c he must set to work to clean the horse, which he ought to have done before instead of going to sleep." A boy going down into the hold of a ship fell from a considerable height, and struck his head. He lay insensible, as it appeared from the observation of his shipmates, about half an hour, when he came upon deck without any assistance. Nevertheless on the following day all the circumstances of the accident had passed from his memory. Some time afterwards when he was received into St. George's Hospital, I found that he knew. nothing of the accident except from the report of others. He had not only entirely forgotten the accident itself, but he did not even remember his having gone down into the hold of the vessel before the accident, nor his having come upon deck afterwards : and he never regained his recollection on these points. Desault mentions the case of a man, who, after a blow on the head, at first had no recollection except of recent events : but afterwards a change took place, in consequence of 
which his memory failed him as to recent events, while he could remember those which had occurred in childhood.

A number of circumstances which it is unnecessary to enumerate, as every physiologist is well acquainted with them, tend to shew that the influence of the brain is by no means necessary to the action of the heart: which may, under certain circumstances, continue uninterrupted, even after the entire removal of the head. Nevertheless, in cases of concussion of the brain, we generally find the circulation more or less affected; the pulse intermitting, irregular, feeble, perhaps scarcely perceptible, and the patient in a state approaching to that of syncope; and such may be his condition for a few minutes, or for the first four or five hours after the infliction of the injury. The connexion and sympathy which exist between the different parts of the nervous system, afford a reasonable explanation of this apparent anomaly, which, however remarkable it may be, is not more remarkable than the syncope which not unfrequently follows the first introduction of a bougie into the urethra, or that which is the consequence of many other trifling injuries of parts remote from the centre of the circulation, and exercising no direct influence over the functions of the heart.

In those cases in which concussion proves fatal it appears to be this disturbance of the- heart's acvol. XIV.

A A 
tion which is the immediate cause of death. In general when the patient has lain for some time in the state which has been described, a reaction of the circulating system takes place, and the pulse beats with greater strength in proportion as the failure of it was greater in the first instance. But where the shock has been unusually severe there is no such reaction. The pulse becomes more and more feeble, more irregular and intermittent; the extremities grow cold, and at last the action of the heart being altogether suspended, the patient expires. In some cases, even after reaction has begun to take place, it seems as if the constitution was unequal to the effort: there is another failure of the circulation, the result of which is the same as if the patient had never rallied from the beginning.

Sect. 4.-Compression of the Brain.

If the dimensions of the cavity of the cranium be suddenly diminished, as in a case of fracture with depression of bone, or if the actual quantity of the contents of the cranium be increased, as in a case of ruptured vessel and extravasation of blood, the functions of the brain become impaired. This is a matter of experience and observation, about which there is no dispute. There may be, however, some difference of opinion as to the physiological explanation of the phænomena which arise in such cases. It has 
been usually held that the substance of the brain is actually compressed; but Mr. Bell observes very truly that we have no more right to believe that the substance of the brain admits of being compressed, than that water is compressible; and he infers, that what is called compression of the brain, operates not on the substance of the brain itself, but simply on its blood-vessels; lessening their diameter, and thus preventing that due supply of scarlet arterial blood which is necessary to a due performance of the vital functions. It is evident indeed that the effect which compression of the brain produces on its vessels must be to a greater or less extent such as Mr. Bell has described it to be. It may, however, be urged on the other hand, first, that in some cases symptoms similar to those which arise from compression, take place where there is a preternatural determination of blood to the head; where the vessels instead of being empty are actually overloaded; and that in these cases the symptoms are relieved by drawing blood from the jugular vein, or from the veins of the arm; as if the pressure occasioned by too much blood in the vessels was productive of nearly the same effects on the brain, with that arising from blood in a state of extravasation: secondly, that, although we admit the substance of the brain to be incapable of being compressed into a smaller compass, yet that the effect of all pressure on it must be, and is, to alter the position 
and relative situation of the delicate fibres of which its minute structure is composed, and that we need seek no further explanation of the symptoms which are met with in these cases.

In whatever way compression of the brain operates so as to disturb the functions of that organ, it is difficult to explain wherefore the symptoms to which it gives rise are sometimes slight, and at other times urgent, although occurring under circumstances apparently similar. A depression of bone, which in one instance produces comparatively little effect, in another case occasions a manifest destruction of sensibility : and the same observation may be made respecting internal extravasations of blood. Every practical surgeon must have observed that there are differences in the symptoms produced, which are not to be accounted for by any difference in the quantity of pressure, nor in the particular part of the brain which is affected by it. At the same time it is undoubtedly true, that, for the most part, the patient suffers more from an extensive than from a slight depression; more from a large than from a small extravasation. There is reason to believe that pressure is on the whole more dangerous when it affects the lower part of the brain, than when it affects the upper part ; and it has appeared to me that more urgent symptoms are produced by a given quantity of blood, when it is effused 
into the cells between the tunica arachnoides and pia mater, than when it is collected in one mass so as to produce a less general pressure.

Having made these preliminary observations, I shall proceed to consider the particular symptoms which arise from pressure on the brain.

1. Pain in the head:-The blow which occasions a fracture and depression of the cranium, or an extravasation of blood within the cranium, is likely to produce concussion of the brain also, and as pain in the head is a symptom of the latter injury, it may be a question, in many instances, to which of these two causes it is to be attributed. That intense pain in the head may, however, be wholly dependent on pressure on the brain is proved by a case in which a patient under my care laboured under this symptom, and no other, except indeed that the pupil of one eye was preternaturally dilated. There was a fracture with depression of a very small portion of one parietal bone, and immediately on the depression being elevated, the pain in the head was completely relieved.

2. Insensibility:-which is sometimes incomplete, corresponding to what is observed in cases of concussion of the brain; the patient lying for the most part unconscious of what passes around him, but capable of being roused by stronger impressions on his senses; while at other times the 
loss of sense is perfect, so that the skin may be pinched, the flame of a candle may be held close to the eye, and the loudest voice may be uttered in the ear, without any evident effect being produced on the sensorium. Where the cause' of these symptoms is a fracture and depression of bone, they shew themselves immediately after the infliction of the injury; but where they depend on an extravasation of blood, as, in many instances, the extravasation may take place slowly, so an interval of time, an hour for example, may elapse before the patient becomes insensible. Not unfrequently there is insensibility, from concussion of the brain in the first instance; then the patient recovers, and afterwards, as the blood is gradually effused within the cranium, he relapses into his former state of insensibility. These observations were made first by Le Dran, and afterwards by Mr. Pott, and it is needless to remark how great is their importance, as connected with the diagnosis of these different kinds of injury. But even when pressure on the brain is actually established, the insensibility to which it gives rise is liable to some degree of variation. At one time it may be perfect; then the patient may shew some signs of consciousness, and then relapse into a state of perfect stupor. It may be observed, that there is especially an increase of sensibility after blood. letting, and that as the effect, which the loss of blood has produced on the circulation, subsides, so the sensibility becomes again diminished. 
If these observations be correct, it is evident that there is not any such difference in the character of the insensibility produced by concussion; and that produced by compression of the brain, as will enable us at once, and in all cases, to distinguish these two kinds of injury from each other. Those who are led to take a different view of the subject, may indeed urge, that in some cases there is considerable pressure on the brain, without any symptoms at all ; and that when, in a case of fracture, and depression of the cranium, or extravasation of blood within the cranium, the patient lies with a partial loss of sense, this is to be attributed not to the actual pressure, but to the concussion of the brain, which the violence inflicted must necessarily have occasioned in a greater or less degree. I might however refer to several cases, to which this explanation cannot be well applied; but a single example will be sufficient. A woman received a blow on the head; after which she was able to walk home, complaining that her head was hurt, and that she had received her death blow. In an hour after the accident, she gradually became insensible. About fourteen hours afterwards she was brought to St. George's Hospital, labouring under symptoms precisely corresponding to those which have been described by Mr. Abernethy, as arising from concussion. These symptoms continued, and even rather abated than increased, until the third day, when an aggravation of them took place, and she expired. On ex- 
amining the body, eight ounces of blood were found effused underneath the dura mater. The circumstance of there having been no loss of sense in the first instance, and the interval of an hour which elapsed between the period of the accident and that of the occurrence of the symptoms, sufficiently demonstrate that they were the consequence of pressure produced by the hæmorrhage, and not of the concussion.

It sometimes happens, that there is a destruction of sensibility in one part of the system, while the general sensibility is impaired only in a slight degree. An old man was admitted into St. George's Hospital, who had been run over by a cart. There was a fracture with depression of one parietal bone. $\mathrm{He}$ was sensible, but slow in giving answers, and peevish, and it was observed that he was totally blind. Mr. Gunning removed a portion of the parietal bone with the trephine, and elevated the depression ; but the operation produced no change in the symptoms. About thirty-six hours after the accident, the pulse became frequent, and he was delirious. He remained entirely deprived of the faculty of vision; believing that he saw imaginary objects, but totally unconscious of the existence of those which were actually before his eyes. At the expiration of the fifth day he died. On examining the body, the membranes of the brain were found to be inflamed, and smeared with pus and lymph. In the basis of the cranium, there 
was a transverse fracture extending across the sphenoidal bone, and the fractured edges were displaced in such a manner as to press on the optic nerves immediately behind the orbits, and to explain, in the most satisfactory way, the total loss of sight. Such cases as that which follows, are not very uncommon. A gentleman was thrown from his horse, and received a blow on the head. He lay with well-marked symptoms of compression of the brain, which however began to subside in a few days. In a short time, his general sensibility was completely restored, but there was a numbness, or loss of sensation, of one hand for more than a year afterwards.

3. Paralysis :-Here, as on other occasions, the same cause which prevents the brain receiving impressions from the nerves, prevents it also transmitting its influence through the nerves to the muscles. Where the destruction of sensibility is complete, the voluntary muscles are completely paralysed. In whatever position the patient may be placed, in that he remains motionless. The bladder incapable of contraction, becomes preternaturally distended with urine ; and the relaxation of the sphincter ani allows the involuntary discharge of fæces from the rectum. Afterwards the muscles of respiration become affected also; the patient breathes with stertor, as in a most profound sleep; and the diaphragm contracts at longer and longer intervals, until respiration altogether ceases. 
It is this paralysis of the muscles of respiration, which in ordinary cases of pressure on the brain is the immediate cause of death. Where there is an imperfect loss of sense, there are often no marks of paralysis whatever. At other times there is paralysis of one side of the body, while the muscles of the other side, continue to obey the will as usual ; and sometimes the paralysis is permanent. Dr. Hennen* gives an account of a patient who recovered with life from the effects of a fracture and depression of the left parietal, and left side of the frontal bone; but fourteen years afterwards, he was still paralytic in the opposite arm and leg.

Hemiplegia is however a much more rare occurrence where pressure on the brain is the consequence of accidental violence, than it is in cases of apoplexy from a spontaneous rupture of a blood. vessel. The difference may reasonably be attributed to the different situation of the pressure. In cases of apoplexy, the extravasation is for the most part situated either in one of the ventricles, or in the substance of the brain; but after a.blow on the head, the cause of pressure more commonly operates on the surface. Occasionally the para. lysis is confined to one set of muscles, or even to a single muscle. There may be, for example, loss of motion in one hand, or a ptosis, or dropping 
down of one upper eyelid. In cases of hemiplegia after an injury of the head, the paralysis is on the side opposite to that on which the pressure exists : at least $I$ have never met with an exception to this general rule. The observation, however, does not apply to more partial paralytic affections. A young gentleman fell from a coachbox, and struck the left side of his head against the wheel of the carriage : he was not stunned, but there was an ecchymosis of the left cheek and temple, a copious discharge of blood from the left ear, and the muscles of the left side of the face were rendered paralytic. When he laughed, the mouth was distorted to the right side; and he was unable to close the left eyelids. The loss of power over the muscles was not attended with any loss of sensation, and was not permanent, the recovery of the patient being complete in about three months. It seems reasonable to conclude that in this case the cause of the paralysis was pressure produced by the extravasation of blood on the portio dura of the nerve of the seventh pair, by which the muscles of the face are supplied, and not on the brain itself. In like manner I have known a ptosis of the left upper eyelid connected with pressure on the inferior surface of the left hemisphere of the cerebrum, the pressure being so situated as to affect the nerve of the third pair im. mediately behind the left cavernous sinus.

4. Convulsive actions of the muscles:-Where 
there is paralysis of one side of the body after an injury of the head, we sometimes observe convulsive twitches of those of the other side. But it appears to me to admit of a question whether this symptom ought to be regarded as the consequence of simple pressure on the brain. We find it occur in cases of punctured and wounded brain, where there is no pressure; and it so happens, where it has fallen under my observation in cases of de. pression of bone or extravasated blood, and where the exact nature of the injury was afterwards ascertained, that the pressure has been always found to be complicated with wound or laceration of the substance of the brain.

The convulsive twitches to which I here allude are slight and only partial, and are to be distinguished from those violent fits of general convulsions on which I shall have to offer some observations hereafter.

5. Affections of the pupils:- The state of the pupils varies very much in cases of pressure on the brain even under circumstances apparently similar. I have seen the pupils dilate with the absence, and contract with the presence of light, although the patient lay in a state of complete insensibility, and did not seem to be at all conscious of the impressions made on the retina. But this is a rare occurrence, and for the most part where the other symptoms of pressure are present, 
the pupils are insensible and motionless; being generally dilated, but sometimes contracted. It is not uncommon for the pupils to remain for a time in a state of dilatation, then to become suddenly contracted, and after remaining so for a longer or shorter time, to become again dilated, these changes taking place independently of light and darkness. 1 have observed especially, where the pupils have been dilated, that they have frequently become contracted immediately after the abstraction of blood; the dilatation returning as soon as the immediate effect of the blood-letting on the circula. tion has ceased. Dr. Hennen mentions a case in which blood was extravasated among the membranes of the brain, and in which the pupils were observed sometimes to become dilated with an increase, and to contract with a diminution of light. In a patient in St. George's Hospital, in whom there was an extravasation of blood on the upper part of the right hemisphere of the cerebrum, and no cause of pressure elsewhere, both pupils were insensible and motionless; but the right pupil was in a state of dilatation, and the left in a state of contraction. In another patient, in whom there was fracture and depression of the left parietal bone, the left pupil was permanently dilated, the right pupil being in a natural state. In a third case, in which there was a fracture and depression of the frontal bone above the right superciliary ridge, there was a dilatation of the pupil of the left eye; and again, in a fourth case, where there 
was a fracture and depression in the same situation as in the case last mentioned, and no cause of pressure elsewhere, both pupils were dilated and equally insensible, but immediately regained their sensibility and power of contraction on the depression being elevated.

As there may be general insensibility without the pupils being insensible to light, so there may be insensibility of one of the pupils without general insensibility, and even without loss of vision. A gentleman fell from his horse, received a severe contusion of the head, and was taken home, labouring under manifest symptoms of pressure on the brain. When, after the lapse of several days, these symptoms became somewhat abated, it was. observed that the pupil of the right eye was dilated, and incapable of contraction; but his power of vision was unaffected. This symptom was accompanied with a ptosis of the right upper eyelid, and a numbness of the right hand. I believe that nearly a year elapsed before the pupil was restored to its natural condition.

6. Affection of the Circulation:-If concussion of the brain be capable of disturbing the action of the heart, it is not remarkable that the greater injury arising from pressure should produce its effect on the circulation also. The effect however is not constant; and sometimes even where the other symptoms of pressure exist, there is no alteration 
of the pulse. Mr. Abernethy has observed that intermission of the pulse is a less frequent occurrence in cases of compression than in those of concussion of the brain. However that may be, I believe it will be found that pressure on the brain for the most part affects the action of the heart; not by producing actual interruption, but by causing its contractions to be either less frequent, or less forcible than natural. The influence of pressure on the brain on the circulation is sometimes very manifest in cases of depression of the bone of the cranium, where the depression is relieved by an operation. A child, three years of age, was admitted into St. George's Hospital having an extensive fracture of one parietal bone, extending into the adjoining portions of the tem. poral and occipital bones. Towards the posterior part of the parietal bone there was a considerable depression, with laceration of the membranes of the brain and of the brain itself. I assisted Mr. Gunning in an operation in which be removed a portion of the bone with a saw, and elevated the depression. Previously to the operation the pulse at the wrist was barely perceptible, but immediately afterwards it became distinct, and beat with considerable strength. A gentleman who held the child's hand during the operation observed the pulse to be suddenly restored at the very instant of the depression being elevated. Another patient (a man) was admitted into the hospital having a fracture with depression of the right 
side of the frontal bone extending into the right parietal. The pulse beat no more than forty times in a minute, but immediately on the depressed bone being elevated it rose to sixty in a minute.

7. Sickness and vomiting:-These symptoms occur in some cases of pressure on the brain from injury, but it may nevertheless admit of a question whether they should or should not be referred to the actual pressure. The same injury which occasions a fracture and depression of the cranium, or an extravasation of blood within the cranium, is likely to produce concussion of the brain also. In cases where the symptoms of pressure are the most distinct, and there is complete insensibility, there is no disposition to vomit; and where I have had occasion to apply the trephine on account of a fracture and depression, and there was no sickness previously, I have sometimes known the patient to become sick and vomit immediately on the depression being elevated.

The symptoms of pressure on the brain vary in different cases, not merely as they may exist in different degrees, but as they happen to be variously combined with each other. We find also that there is a great difference as to the period of their duration. Of two individuals, in whom the 
early symptoms appear to be equally urgent, one may die in the course of three or four hours, and another may survive for several days; and among those who recover, we may find some in whom the symptoms wholly subside in the course of a few days, and others in whom some remains of them exist after the lapse of several months, or even of years. Even in fatal cases the symptoms are not in every instance uniformly progressive, and it is not very unusual for them in some degree to subside, recurring afterwards with increased severity.

Where blood-vessels have been ruptured or wounded in other situations, secondary hæmorrhage occurs in some instances at the end of a few days from the period of the injury having been inflicted. Does secondary hæmorrhage ever occur within the cavity of the cranium? In one case, which came under my observation, I was led to believe that this actually happened, causing sudden death after three or four days of apparent convalescence. As I have met with no other instance of the kind, I conclude that such occurrence is very rare; but probably it would be more frequent, if it were not that in the practice of modern surgery a very strict antiphlogistic regimen is usually pursued for a considerable time after the occurrence of the accident. The following is a brief outline of the case to which $I$ allude. 
A man, thirty-five years of age, on the afternoon of the 8th of November, fell from a cart and struck his head against the pavement. A medical practitioner in the neighbourhood bled him, and he was afterwards brought to St. George's Hospital talking and reeling like a drunken man. He was again bled. On the following day he complained of head-ach, but was otherwise well. He continued without any symptoms until five in the morning of the 12th of November, when some of the patients in the same ward heard him talking incoherently. The nurse called the house surgeon to him, but before he could arrive the man had become insensible, and was found lying motionless with stertorous respiration and dilated pupils. Blood was taken from the arm, but the symptoms were not relieved, and he died in about half an hour after the commencement of the attack. On examining the contents of the cranium after death, a thin layer of blood was found extravasated in the cells between the tunica arachnoides and pia mater, where those membranes cover the posterior part of the two hemispheres of the cerebrum. In the lower part of the right anterior lobe of the cerebrum the substance of the brain had been ruptured, and underneath this part, between the dura mater and tunica arachnoides, there was a collection of about two ounces and a half of blood. This last had all the appearance of a recent extravasation, and seemed to 
afford a satisfactory explanation of the sudden alteration in the symptoms which immediately preceded the patient's dissolution: the hæmorrhage in the first instance having in all probability been checked by the blood-letting which was resorted to both immediately after the accident, and on his admission into the hospital.

SECT. 5.-Wounds of the Brain and its Membranes.

Wounds of the dura mater, greatly as they aggravate the ultimate danger of the case, do not in themselves add to the symptoms which immediately follow the accident. It is when the period of inflammation has arrived, and not until then, that the marks of punctured or lacerated dura mater show themselves.

The pia mater and tunica arachnoides are so thin and delicate in their structure, and so intimately connected with each other, and with the brain itself, that we cannot conceive them to be wounded without the brain being wounded also. It would be idle therefore to treat of these two classes of injury as being distinct from each other.

The researches of modern science have demonstrated that the brain is composed of various organs, intended to exercise very different functions: and the division of the substance of the brain madeby the hand of the physiologist produces 
very different effects, accordingly as it detaches one or another of these organs from the rest of the nervous system. But those distinct results which are obtained with difficulty in experimental physiology, are not met with in cases of accidental wounds. The symptoms produced by the latter are always liable to be complicated with those of concussion, and in a great number of instances are also complicated with those of compression of the brain. Accidental wounds rarely affect the cerebellum and medulla oblongata, or even the more deep-seated and important parts of the cerebrum: and with respect to wounds of the cerebrum, such as are commonly met with, even without the complications produced by concussion, or depression of bone or extravasated blood, we find their effects to be so different in different cases, that they do not admit of being reduced to any general rule; and no data, which we have hitherto obtained, will enable us to predict the exact consequences to be produced by a wound of a given extent, or occurring in a given situation.

In illustration of this observation I may refer to two cases, related, the one by Morgagni *, the other by Dr. Hennen $t$. In the first of these cases a man received a punctured wound from a sharp instrument, which passed between the eye and the roof of the orbit, penetrating through the latter

* Letter 51. a. 57. † Military Surgery, p. 286. 
into the substance of the cerebrum to within a finger's breadth of the lateral ventricle. In the second case the extremity of an iron ramrod entered the cranium immediately below the nasal process of the frontal bone, and penetrated one inch into the anterior. lobe of one hemisphere of the cerebrum. In each of these cases the wound was of the same kind, and very nearly in the same situation : but in one of them it was considerably deeper than it was in the other. It might well be supposed that there would have been some correspondence in the effects produced:-but what were the actual results? In Dr. Hennen's case, where the injury was the slightest, the patient was instantaneously deprived of life: while in Morgagni's case, where the injury was greatest, there were no symptoms whatever, and the patient was as if nothing unusual had occurred until the end of the third day, when suppuration was established.

Of these two cases, however, it must be allowed that the latter is to be regarded as being more in accordance with the general rule than the former. The experience of every individual who has had the opportunity of seeing many cases of injury of the head will afford examples of wounds penetrating into the substance of the brain, as well as of incised and lacerated wounds, in which the functions of the brain were not at all impaired, or only slightly impaired in the first instance. . Even actual loss of the substance of the brain not un- 
fiequently takes place without the occurrence of any urgent symptoms, and the patient may go on from day to day, with fresh portions of the brain oozing out of the aperture in the cranium, with his external senses perfect, his mental functions unimpaired, and fiee from paralytic affection.

It is not however to be supposed that there can be an extensive destruction of a part so important as the brain, without immediate death, or death in the course of a very few hours. In other cases in which the brain has been extensively lacerated, it has appeared to me that without the actual insensibility which follows concussion of the brain, there was a confusion of intellect beyond that which concussion usually produces. In many cases of wounded brain there are convulsive twitches of the muscles of the extremities. In a case in which there was fracture of the parietal bone, several splinters of bone having been driven into the substance of the cerebrum, on the splinters being removed, and when no evident cause of mischief remained except the wound which they had occasioned, the pupil of the eye of the opposite side remained preternaturally dilated. This is what might have occurred in consequence of pressure on the brain. It corresponds also to what we observe in cases of pressure, that wounds of the brain sometimes occasion an unnatural slowness of the pulse. But the more urgent symptoms of pressure are wanting; and the peculiar danger of wounds 
of the brain arises, in the great majority of instances, not from the immediate effects of the injury, but from the extensive and intractable inflammation which takes place afterwards.

Sест. 6.-On some other Symptoms following. Injuries of the Brain.

The symptoms of which I propose to give an account in the present section also belong to the class of those which immediately follow an injury of the brain, that is, which shew themselves previous to the occurrence of inflammation. I have however thought it better to give them a separate consideration, because there may be some doubts as to the exact nature of the injury of which these symptoms are to be regarded as the indication, and because there are several points respecting them which require to be elucidated by further observations.

I. A middle-aged man received a blow on the head and was brought to the hospital with symptoms which were supposed to arise from concussion of the brain. These symptoms subsided in the course of one or two hours, but he remained afterwards completely deaf. His relations declared that his hearing had been perfect up to the period of the accident. He left the hospital at the expiration of three weeks without the smallest amendment. 
A young woman received a blow on the head, by which she was stunned for a few minutes. After she recovered from the immediate effects of the accident, she found herself entirely deprived of the senses of smell and taste, and she was in this.state when I saw her a month afterwards. The strongest and most pungent odours produced not the slightest sensation when applied to the nostrils; but they nevertheless increased the secretion of the lachrymal glands, or in common language made the eyes water, as under ordinary circumstances.

A middle-aged man slipped while walking, and struck the back of his head against the road; he was stunned for five or six minutes, then recovered so as to walk home. He saw objects double during that evening, and it was observed that he was deaf in one ear. He was kept awake by violent headach during the night. On the following day he had recovered from the double vision, but the other symptoms continued, and in addition to them he discovered that he had entirely lost his sense of smell, and that there was also a partial loss of the sense of taste. He was bled several times, and kept on a low diet, and under this treatment the headach gradually subsided, and at the end of about four months he had recovered his sense of hearing. When he consulted me between five and six months after the occurrence of the accident, he was in the following condition. 
His pulse was 72 in a minute. He complained of a sense of noise in the right side of the head, especially in the morning and evening, but not during the night. He was impatient and irritable, especially when troubled respecting matters of business. He had no proper sense of smell, common odours were not perceived at all; but he felt the pungency of smelling salts, and they made his eyes water. With his taste he could distinguish bitter, sweet, and sour, but he was unable to distinguish flavours accurately. For example, he could perceive a difference between the taste of hops and that of sugar, but not between that of fennell and parsley; and the flavour of game was the same to him as that of other meat. Bitters had become disagreeable to him, though they had not been so formerly.

The late Mr. Grover of Hammersmith informed me of the case of a gentleman who had been under his care on account of an injury of his head, which entirely deprived him of the sense of smell. After some time, however, he began to recover of this symptom, and at the end of a year his smell was completely restored. I have already given an account of a case in which an injury of the head was followed by total blindness with permanent dilatation of the pupils, and this was found to have depended on a fracture and displacement of the bone in the basis of the cranium producing 
pressure on the optic nerves. But here there were other symptoms manifestly depending on compression of the brain itself : whereas no'such symptoms existed in the cases which I have just related. It is indeed difficult to conceive that pressure on the brain should exist in so great a degree as completely to destroy an entire class of sensations, and at the same time be so partial as not to affect any other function of the nervous system. On the other hand it is also difficult to regard these as the effects of concussion of the brain: since it is one of the characteristics of concussion to produce no more than a diminution of sensibility, and that diminution, instead of continuing for months or.years, is completely relieved in the course of a few days, and probably in a much shorter space of time. However produced, these are not the only examples which experience affords of partial nervous affections following an injury of the brain. Dr. Hennen gives the history of a patient who lost his sexual powers after a wound of the occiput. The same author observes, "The powers of speech are often lost while those of memory remain, and the sight is impaired while the hearing is perfect, and vice versâ. I have met with numerous instances of this, and have had patients who told me that they could hear distinctly what I said, and distinguish my voice from that of others, and have repeated my words as a proof both of this fact, and of their retention of 
memory, while they could not distinguish my person or give utterance to their thoughts." *

II. In some cases after an injury of the brain we find the patient attacked by violent convulsions affecting the whole person, and entirely different from those slight involuntary twitches of the muscles which have been already noticed. These convulsions a good deal resemble those which constitute a fit of epilepsy, but are not, like the latter, uniformly followed by a state of profound sleep or stupor. They are more formidable in appearance than in reality, as it is not uncommon for the patient after the convulsions have subsided to recover without any unfavourable symptoms. A young man, a butcher, was standing under a beam of wood which supported a side of beef, when the beam gave way and fell. The side of beef came obliquely on his back, and the beam by which it was supported struck his head. He was not immediately stunned, but in about a minute he became insensible, and in ten minutes more he was seized with a fit, in which he was violently convulsed, so that four or five persons were required to hold him. He was bled, but without relief. The fit of convulsions lasted for nearly three hours, and then suddenly left him. He now complained of pain in the head, but was perfectly sensible. He recovered without any further symptoms, except 
that the pain in the head continued, and on this account he was bled twice or three times in the course of the ensuing week or ten days.

A gentleman on the 8th of September, 1825, was thrown from his horse, and falling on the pavement received a blow on the arm which occasioned a fracture communicating with the elbow joint, and another blow which caused the scalp to be separated for a considerable extent from the anterior part of the head, and also occasioned a fracture of the frontal bone, but without depression. He was taken up in a state of insensibility. He was in this state a few minutes afterwards, when he was seized with violent convulsions, his limbs being moved in various directions, and with such force, that it was with much difficulty that several persons could hold him. The convulsions continued for about half an hour, when they subsided, leaving him in a state of stupor. Blood was now taken from his arm, after which he began to regain his sensibility. On the following day his sensibility was completely restored, and he recovered without any further unfavourable symptoms.

In these cases the convulsions took place within a short period after the occurrence of the accident; but there are others in which the patient is affected in the same manner, after the lapse of several days. Here the convulsions must often be combined with symptoms of inflammation, so that 
it may be difficult to determine whether they are to be regarded as connected with the original mischief produced by the injury, or as arising from the subsequent inflammation. The following case however seems to prove that in some instances at least the convulsions which occur even at this second period depend on the former cause and not on the latter.

A lad, 14 years of age, received a blow on the head, and became instantly insensible. He did not utter an intelligible word, nor could he be prevailed on to show his tongue, nor to swallow either medicine or the liquid nourishment which was offered to him. However, he moaned when disturbed, the pupils of his eyes were sensible to the stimulus of light, and there was neither stertor nor paralysis. These symptoms slowly subsided, and no new symptoms, such as could be regarded as the result of inflammation, had shewn themselves, when at the expiration of five days after the accident he was seized with convulsions agitating his whole person. Blood was taken from him by cupping, but this afforded no relief, and in the course of the succeeding twenty-four hours he had as many as fourteen or fifteen attacks, each lasting from one to three minutes. On the following day the state of the pulse not being such as to indicate the necessity of the further abstraction of blood, I determined to pursue an opposite plan of treat- 
ment. He was prevailed on to take beef-tea with toast; this was repeated at short intervals, and from the time of his beginning to take more nourishment the convulsions abated, and in the course of an. other day had wholly ceased. From this time his recovery proceeded uniformly and favourably.

In two of the cases which have been just related the other symptoms were such as might have arisen, and probably did arise, merely from concussion of the brain. This however does not prove the entire absence of extravasation, and there are some circumstances which may lead to the suspicion that something more than concussion is necessary to produce such attacks of convulsions as those which have been described, and which at any rate shew that they may arise from other causes.

First, I have observed in experiments on animals that a wound on the basis of the brain which causes extravasation of blood on the surface of that organ, generally produces convulsions previous to that state of stupor and paralysis which immediately precedes death.

Secondly, the ordinary symptoms of concussion occur, and indeed are more complete, immediately after the injury is inflicted than at any subsequent period; whereas, according to my ex- 
perience, convulsions never occur until after a certain lapse of time, when extravasation may have begun to take place.

Thirdly, the following case occurred in St. George's Hospital, under the care of Mr. Keate. A man was admitted who had fallen from the top of a coach, and had struck his head. He was stunned, and continued insensible after being brought to the hospital. At the end of two days, when he had begun to recover from this state of stupor, he was seized with violent convulsions, affecting not only the muscles of his limbs, but also those of his face. The first attack of convulsions continued about six minutes, but this was succeeded in the course of an hour and a quarter by four similar attacks, and in spite of a considerable quantity of blood being taken from the arm. At the end of this time Mr. Keate saw him, and made an incision through the scalp at that part which had received the violence of the injury. A fracture about an inch in length was discovered at the posterior part of the left parietal bone, extending into the lambdoidal suture with a slight depression. At this part Mr. Keate applied a saw, and removed the depressed portion of bone. A small coagulum of blood was found lying on the surface of the dura mater, and this having been exposed there was no recurrence of the convulsions.

I have not observed convulsions to take place 
where there are symptoms indicating the existence of considerable pressure on the brain. The pressure in these cases does not destroy the functions of the brain; it seems to act merely as a cause of irritation, and the operation of it may be compared to that of an exostosis, or other tumor, in producing fits of epilepsy. 'The circumstance of convulsions taking place after the lapse of some days when they did not take place in the first instance, may probably depend on the brain having been rendered more susceptible by the loss of blood and other methods of depletion to which it was necessary to have recourse for the relief of the more early symptoms.

III. Occasionally after an injury of the head we find the patient in a state of furious delirium, raving and unmanageable. A man who had received a blow on the head was brought into St. George's Hospital in this condition, uttering loud exclamations, abusing and striking those who were near him, so that it was necessary for several persons to assist in holding him by force as if he were a maniac, while blood was being taken from his arm. As the blood flowed the delirium left him. He remained with slight symptoms of concussion; and these also gradually subsided, leaving the patient in a state of health. Cases such as this might lead us to regard this state of furious delirium as the consequence of mere concussion of the brain. But the same observations may be made respecting these 
cases, as respecting those in which there are convulsions. The absence of the more urgent symptoms of pressure on the brain does not absolutely prove that no degree of pressure actually exists : and instances occur in which this state of the sensorium is manifestly combined with depression of bone or extravasated blood. For example :A middle aged man, who had received a blow on the head, was brought to St. George's Hospital an hour after the occurrence of the accident, in a state of raving delirium. There was a wound over the right eye-brow, and a fracture of the frontal bone extending obliguely upwards with a considerable depression. The depression, however, was not elevated, as the delirium subsided on blood being taken from the arm. After this the man fell into a state of insensibility, from which, however, he could be roused, and then he complained of head-ach. On the following day he was more sensible, and from this period he recovered without any bad symptoms; but it was observed that the pupil of the right eye remained preternaturally dilated, and that it contracted very feebly on exposure to light.

A middle aged man fell from a cart, and struck his head against the wheel. In about half an hour he was brought to St. George's Hospital. He was sensible, and complained of pain in the head, but more of pain in one arm, which was discovered to have been fractured. At this time he had no 
other symptom except that the right pupil was more dilated than the left. There was a wound of the scalp and a fracture with a slight depression of the anterior and inferior part of the left parietal bone. He was put to bed, and while his head was being shaved he became delirious, furious, and unmanageable, so that it was necessary to restrain him by main force. On being bled, he became faint, tranquil, but not perfectly sensible. In half an hour the faintness had subsided, and he relapsed into his former state of raving delirium. He was again bled, and became more tranquil, but still not perfectly sensible. In the evening, twelve hours after his admission, as he continued insensible, Mr. Gunning applied the trephine in the situation of the fracture, and removed a portion of bone. The man appeared to be relieved, and spoke rationally after the operation. On the following day he was quiet, and sensible when roused, but not so to ordinary impressions. Early on the next morning he fell into a state of stupor, with stertorous breathing, a slow pulse, and cold extremities, and soon afterwards expired. On dissection there was discovered a disjunction of the coronal suture in some degree separating the parietal and frontal bones from each other. From a drachm to a drachm and a half of blood was extravasated be:: tween the dura mater and the right side of the frontal bone, and the right parietal bone. There was also in some parts a slight degree of extravasation in the cells between the tunica arachnoides. 
and pia mater. A small quantity of pus was found both between the dura mater and the bone, and between the tunica arachnoides and pia mater.

In another case where the patient was admitted into the hospital with the same symptoms of furious delirium, after the delirium had subsided he fell into a state of perfect stupor, from which he could not be ronsed until twenty ounces of blood had been taken from the arm: and when the immediate effects of the blood-letting had subsided he again relapsed into the same state of stupor. The pupil of one eye was observed to be preternaturally dilated, contracting in some degree, but imperfectly, on exposure to light. This patient ultimately recovered, and of course it was not possible to be made acquainted with the exact nature of the injury which he had sustained, but I was led to regard the state of complete insensibility in which he for some time lay, joined with the dilatation of one pupil, as a sufficient indication of the existence of pressure on the brain to a greater or less extent.

From the evidence here adduced there seems reason to believe that furious delirium and convulsions occur after an injury of the head under nearly parallel circumstances. The former symptom, like the latter, may be produced by pressure on the brain, not however by such a degree of pressure as threatens completely to anmihilate the 
function of that organ, but by that smaller degree of pressure which operates merely as a source of irritation. It must be admitted, however, that the subject is not exhausted, and that further observations are required for its complete elucidation.

SEcr. 7.-Treatment of concussion of the Brain.

Although the treatment which is required in the first period which elapses after an injury of the head is neither various nor complicated, yet, in order that it should be conducted with advantage, it is necessary that many circumstances should be taken into consideration. We are called upon not only to do that which is to contribute to the relief of the present symptoms, but to guard against future ill consequences, and where no symptoms actually exist we are to look to those which may occur hereafter, and which proper measures of precaution may enable us to prevent or mitigate.

It is commonly remarked that two opposite methods of treatment have been recommended in cases of concussion of the brain ; the one consisting of the exhibition of stimulants and cordials: the other comprising blood-letting, and what are usually termed antiphlogistic remedies. Here, however, as on many other occasions, the oppo- 
sition of opinion is probably greater in appearance than in reality; and I am inclined to believe that if the advocates of the respective systems were questioned on the subject, it would be found that the views which they entertain are not essentially disșimilar. I suppose that none of those who have suggested the exhibition of stimulants would actually be inclined to apply this practice to cases in which the pulse has regained its strength and regularity; and, on the other hand, I conclude that no one among those who have advised the use of the lancet would think of taking away blood when the patient lies with pale cheeks, and cold extremities, and a feeble and intermitting pulse, or would refuse to resort to the cautious exhibition of cordials and stimulants where these symptoms are so urgent that he is manifestly in danger of sinking, in consequence of the depressed state of the circulation which has followed the first shock of the injury.

Cases of this last description are however in reality of rare occurrence: and there are indeed sufficient reasons why we should regard that condition of the system which approaches to syncope, as being, in the great majority of instances in which it exists, conducive to the patient's welfare, and why we should wish to prolong, rather than to abridge, the period of its duration. The same blow which gives rise to symptoms of concussion frequently occasions the rupture of some small 
vessels within the cranium. The same state of the system which produces an enfeebled action of the heart is calculated to prevent the ruptured vessels from pouring out their contents; and the longer it continues, the less is the danger of internal hæmorrhage. If we artificially excite the action of the heart by the exhibition of wine and anmonia, we are in danger of inducing symptoms of pressure on the brain. If on the contrary we watch the gradual restoration of the pulse, and at the proper moment take from the arm a sufficient quantity of blood to prevent the heart resuming its wonted action, it is probable that we may often succeed in checking or arresting an extravasation of blood on the surface of the brain, or among its membranes, which might otherwise prove fatal. There is also the following very important circumstance which is not to be overlooked in this part of the inquiry. A state of depression is followed by a state of excitement. As the patient recovers from the former, the pulse, with respect to fulness and strength, becomes raised above the natural standard, and it is evident that this affords an additional argument in favour of the practice which is here recommended.

The same views respecting the prevention of internal hæmorrhage, which incline us to take blood from the arm in the first instance, cannot fail to influence our conduct afterwards. There is no evident reason why vessels, which have 
once bled, should not be liable to bleed again within the cranium, as well as in other situations. I have already mentioned a case in which a patient, who was apparently going on favourably, suddenly expired in consequence of such secondary hæmorrhage, on the fourth day after the occurrence of the injury. If similar cases are rare, this may reasonably be attributed to the remedies which modern surgeons, with few exceptions, do not fail to employ. At any rate, where so much is at stake, we are called upon to neglect no measures of precaution; and however small the danger from this cause may really be, the surgeon should provide against it, by frequently inquiring into the state of his patient : by urging the necessity of continued repose of body and mind, by limiting him to a scanty vegetable diet, by the exhibition of laxative medicine, and by the ab. straction of blood, whenever the state of the pulse indicates that this may be done with propriety.

Independently of the foregoing, there are other considerations which might of themselves lead us to adopt the same method of treatment. I believe that the patient in cases of concussion will generally spontaneously recover from that state of insensibility in which he remains after the vigour of the circulation is restored. But, nevertheless, from the best observations which $I$ have made on the subject, I cannot doubt that his recovery is much assisted by repose and low diet, and de- 
pleting remedies. Often, immediately after being bled, the patient, who before waş in a state of stupor, exhibits manifest signs of returning sense. Further, it may be urged that concussion is liable to be followed by inflammation of the brain, or its membranes. Now I do not mean to say that such inflammation can always be prevented, or that the abstraction of very large quantities of blood will make the patient a better subject for it if it should occur; but it seems reasonable to suppose, and our experience of these cases, and of other cases bearing an analogy to them, confirms the opinion, that there is less danger of inflammation, where the antiphlogistic treatment has been carried to a moderate extent, and where the patient has been kept in a state of perfect quiet, than where bleeding and laxative medicines have been neglected, and the patient has been allowed to exercise his body and mind, and to live on his usual diet.

The quantity of blood which the vessels of the brain contain depends very much on the position of the head with respect to the rest of the body. Not only in cases of concussion, but in all other cases where there has been an injury of the brain, or one likely to affect the brain, the head and shoulders should be raised by additional pillows, so that the blood may have an easy descent to the right side of the heart. In addition to this, in severe cases of concussion, the head should be shaved, and compresses should be applied con- 
stantly moistened with a cold evaporating lotion. Opiates should be avoided. It is difficult to conceive what good purpose they can ever have been expected to answer; and, at any rate, they tend to constipate the bowels, and not unfrequently cause a confusion of symptoms, the patient complaining of head-ach, of which it is difficult to say whether it belongs to the injury itself or to the opium.

In taking a view of the various satisfactory reasons which may be urged in favour of a particular plan of treatment in cases of concussion of the brain, we must not overlook the circumstance that this treatment may be carried too far: and we must eindeavour to avoid the error which I have known some surgeons fall into, of resorting to a too free use of the lancet. At first when the reaction of the heart has taken place, it may be right that the patient should lose a considerable quantity of blood, so as completely to subdue the force of the circulation. Afterwards, for the most part, it is only an occasional blood-letting that is required, and that to a moderate extent. It has appeared to me that this mode of proceeding has usually done more, both towards relieving the present symptoms, and preventing subsequent inflammation, than a more active system of depletion: and where very large quantities of blood have been already taken away, if inflammation should shew itself, our resources are comparatively limited, and 
we are not able to meet it with that energy and vigour which the circumstances of the case require.

Where bleeding has been carried to a great extent, symptoms frequently occur which in reality arise from the loss of blood; but which a superficial observer will be led to attribute to the injury itself, and concerning which indeed it is sometimes difficult, even for the most experienced surgeon, to pronounce in the first instance to which of these two causes they are to be referred. Repeated copious blood-letting is of itself adequate to produce a hardness of the pulse, which we shall in vain.endeavour to subdue by persevering in the same system of treatment. In many individuals it will produce head-ach and confusion of mind, not very different from what the injury itself had previously occasioned. These things may be observed especially in young females who are disposed to hysteria; and whom I have often known to suffer from a continued aggravation of such symptoms as I have described, while the system of depletion has been continued, recovering immediately on the use of the lancet being laid aside, and on their being allowed to take solid nourishment, with occasional doses of the carbonate of ammonia *.

* Dr. Marshall Hall has published, in the thirteenth volume of the Medico-Chirurgical Transactions, some excellent practical observations on the effects of copious blood-letting, many of which are applicable to the cases mentioned above. 
Sect. 8. Treatment to be employed in cases of Compression of the Brain not complicated with Wounds of the Brain or its membranes.

When we consider the variety of circumstances under which compression of the brain may follow an injury of the head, and the different effects which it produces in different instances, we cannot suppose that the same mode of treatment will be found applicable to all cases, or that any such simple rules can be laid down for the conduct of the surgeon as those which we have to guide us in cases of concussion.

There is one most important complication which aggfavates very much the ultimate danger, not only of these, but of all other cases of injury of the head ; namely, the existence of a wound or laceration of the dura mater. This circumstance also tends to modify if not to alter the surgical treatment which is to be adopted. At present I suppose that such a complication does not exist; that the brain suffers from pressure, but that the dura mater is entire, and that there is no exposure of the important parts which are contained within it.

Where the symptoms of compression are such that the patient's life is manifestly in danger, there can be no question as to the propriety of removing 
the cause on which they depend, where that can be accomplished by means of a surgical operation.

In cases in which there is a fracture and depression of bone, it is generally in our power to remove or elevate the depression. If there be a wound of the scalp we may at once resort to the application of the trephine, or in some cases, where the cranium is not only fractured but splin. tered, we may do what is required by means of the forceps and elevator, without the aid of the saw. Where however the scalp remains entire, it will of course in the frrst instance be necessary to divide it, so that the bone may be completely exposed, and that the surgeon may be enabled to trace the extent of the mischief which has been inflicted on it.

An operation is also to be resorted to in those cases in which there are symptoms of pressure depending on hæmorrhage between the dura mater and the bone. But here another question arises : what is the evidence which is to enable us to detect a mass of extravasated blood in this situation, and how are we to determine what is the exact part of the cranium which should be perforated by the trephine? I must here refer to an observation which has been already made. Blood is seldom poured out in any considerable quantity between the dura mater and the bone, except in conse- 
quence of a laceration of the middle meningeal artery, or one of its principal branches, and it is very rare for this accident to occur except as a consequence of fracture. If therefore we find the patient lying in a state of stupor, and on examining the head we discover a fracture with or without depression, extending in the direction of the middle meningeal artery, although the existence of an extravasation on the surface of the dura mater is not thereby reduced to an absolute certainty, it is rendered highly probable, and the surgeon under these circumstances would neglect his duty if he omitted to apply the trephine. If it happens that no extravasation is discovered, the operation does not leave the patient in a worse condition than he was in before : but if there be an extravasation, although it does not place him in a state of absolute security, it relieves the present symptoms, and gives him a chance of recovery which he would not have had otherwise.

Where no fracture is discoverable, yet if there is other evidence of the injury having fallen on that part of the cranium, in which the middle meningeal artery is situated, the use of the trephine may be resorted to on speculation, rather than that the patient should be left to die without an attempt being made for his preservation. I cannot indeed adduce any particular experience of my own in favour of what is here recommended; but I conceive that the instances which 
have been recorded, in which the middle meningeal artery has been ruptured without any fracture of the bone, and the known fact that there is sometimes a fracture of the inner table of the skull, while there is none of the outer table, sufficiently justify such an experiment in desperate cases, or even in those in which there is much danger. Our judgement may be assisted on those occasions by attending to the rule laid down by Mr. Abernethy: "If there be so much blood on the dura mater as materially to derange the functions of the brain, the bone to a certaint extent will no longer receive blood from within; and by the operation performed for its exposure, the pericranium must have been separated from its outside. I believe that a bone so circumstanced will not be found to bleed, and I am certain that it cannot bleed with the same freedom and celerity as it does when the dura mater remains connected. with it."*

In applying the trephine on account of a fracture with depression, the removal of a small portion of bone is generally sufficient; and there is indeed no sufficient reason for removing any considerable portion of the cranium. But in resorting to the application of the trephine, on account of an extravasation of blood on the surface of the durat mater, our practice should be different. The bone

* Abernethy on Injuries of the Head. Edit. 179\%. Pp. 33; 34: 
should be removed extensively, so as to expose at any rate a large portion of the surface of the dura mater, in which the extravasation has taken place. The necessity of attending to this rule, was impressed on my mind by a case which came under my care in the hospital, in the year 1814. A man was admitted with a fracture of the parietal bone, and a large extravasation of blood, between the cranium and the dura mater. I removed two triangular pieces of bone with a straight saw, and a large quantity of blood, partly fluid, partly coagulated, escaped through the opening that was made. The symptoms under which the patient laboured, were immediately relieved, and for several days he appeared to be going on favourably. But suppuration ultimately took place on the surface of the dura mater, wherever the extravasation had separated it from the bone. The opening made by the saw being in great measure occupied by granulations from the dura mater; afforded no opportunity for the free escape of the pus which was formed in the neighbourhood, in consequence of which the abscess burrowed between the dura mater and the bone, separating them from each other, much farther than they had been separated originally. As soon as I had discovered what was taking place, I removed another portion of bone with the trephine; but the mischief had now become so extensive that the operation gave scarcely temporary relief, and the patient died. Reflecting on the case afterwards, 
I could not but acknowledge that if I had removed a larger portion of the bone in the first instance, $s 0$ as to expose the extravasated blood more completely, the pus which was afterwards secreted could have been freely discharged, and the life of the patient would in all probability have been preserved.

But the most common cause of pressure on the brain is an extravasation of blood within the cavity of the dura mater. Here if there be any large collection of blood in one mass, it is generally in the basis of the cranium; sometimes in the substance of the brain, at other times in the cells between the tunica arachnoides and pia mater. In either of these cases it is beyond the reach of an operation. There may indeed be a large extravasation of blood on the superior surface of the cerebrum immediately beneath the dura mater: but if such an extravasation does exist, in what manner are we to become informed of its existence? We may regard it as a general rule, that an operation is not applicable to cases of compression of the brain from internal extravasation. But there are few general rules in surgery, to which some exceptions may not be made. Let us suppose a case in which a considerable portion of bone has been already removed; in which the dura mater is seen exposed, of a blue colour, lifted up by a collection of blood beneath it, and bulging as it were into the aperture, which has been made in 
the cranium. Are we justified in puncturing the dura mater for the purpose of allowing the extravasation to escape? Every thing that we see of wounds of the dura mater tends to prove the very great danger of this kind of injury. The dura mater should never be wantonly punctured; but we cannot doubt that, in what may be regarded as desperate cases, it must be right to give the patient the chance, small as it may be, which the division of the dura mater affords him. The combination of circumstances which would lead to such an operation must be very rare, but it may occur nevertheless, and the surgeon should be prepared to meet it. The late Mr. Chevalier was called to a child a year and a half old, who had received a severe blow on the head. The child lay in a state of insensibility, and was affected with convulsions. There was no wound of the scalp, but on an attentive examination of the head the fontanel appeared to be somewhat elevated. Mr. Chevalier was led therefore to make a crucial incision of the scalp, by dissecting up the corners of which he exposed the fontanel. He then made an angular incision of the right side of the fontanel, and raised the membrane forming it so as to expose the surface of the dura mater, beneath .which the purple colour of extravasated blood was plainly to be seen. A puncture being made carefully with a lancet, the blood issued at first with considerable force spouting to the distance of a foot. Three or four

vOL. XIV.

D D 
ounces of blood escaped; the symptoms were immediately relieved, and the child recovered without any further unfavourable symptoms*.

The following case, which is still more remarkable, was communicated to me by Mr. Ogle of Great Russell Street, in whose practice it occurred some years ago.

A woman, who kept a cellar in Monmouth Street for the sale of second-hand linen, \&c. fell from the street, head foremost, to the bottom of the cellar. When taken up she was in a state of total insensibility. Mr. Ogle being immediately sent for found her lying as if in a fit of apoplexy. He ordered her head to be shaved, and, on examining it afterwards, discovered no wound of the scalp, but observed that she flinched very much when pressure was made on one spot near the anterior and superior angle of one of the parietal bones. Having made an incision of the scalp at this part, he could perceive no appearance of fracture. Nevertheless as the woman was manifestly in imminent danger, he thought it expedient to remove a portion of the bone with the trephine. Immediately on the bone being removed, the dura mater of a dark colour rose into the opening nearly as high as the external surface of the cranium. Convinced from its appearance

* Medical and Physical Journal, Vol. VIII. p. 505. 
and from the feeling of tension communicated to the fingers, that a fluid was interposed between it and the brain, and that that fluid was blood, Mr. Ogle ventured to puncture the dura mater with the point of a lancet. The puncture was instantly followed by a stream or jet of blood, which spirted out to the height of some feet. Immediately on the blood being discharged, the woman, who till that moment had continued totally insensible, opened her eyes. After looking about her, apparently amazed, she exclaimed, "What is the matter? what are you doing with me?" and was able to give a clear account of the manner in which the accident had occurred. From this time she recovered without any untoward symptoms. It was impossible to ascertain the precise quantity of blood which escaped through the opening of the dura mater, but Mr. Ogle supposes it to have been about three quarters of an ounce. But cases such as these are to be regarded as out of the common course of events. The ordinary cases of extravasation within the dura mater from injury are to be treated as we treat cases of apoplexy, or of paralytic seizure, in consequence of a blood-vessel within the head being ruptured from disease: on the same principle as that on which we treat other cases of internal hæmorrhage. Take blood from the arm so as to reduce the force of the heart's action. Repeat this, or take blood by cupping, as soon as the pulse has recovered from the effect of the former blood-letting:-ad-

D D 2 
minister active saline purgatives; let the head be shaved and bathed with a cold lotion, being kept at the same time in an elevated position; and al. though such a plan of treatment will not effect the cure of a patient who lies with stertorous breathing in a state of perfect stupor, many will recover under it, in whom the symptoms of pressure have been very urgent. In some instances a slight improvement is perceptible from day to day, until at the end of two or three weeks the patient seems to be restored to his natural condition. In other instances his recovery is less complete, and a partial loss of nervous power may remain for many months; or such a memorial of the accident as, a dilated pupil, a benumbed hand, or a paralytic limb, may exist for a much longer period, for years, or even during the remainder of the patient's life.

The foregoing observations are intended to relate to those cases in which pressure operates on the brain in such a manner as considerably to impair its functions. There are many other cases in which there is reason to believe that there is extravasation of blood within the cranium, although not in sufficient quantity to produce any formidable symptoms. I have already observed that it is sometimes difficult to distinguish such eases from those of concussion of the brain; and 
if is therefore fortunate that, even where the distinction is plain, it leads to no difference of treatment.

It is also not uncommon for a fracture of the cranium to exist, with even a considerable depression of bone, and for the patient to suffer from it only in a very small degree, or to have no symptoms at all. Mr. Abernethy has published an account of several cases of this description, in which there were not only no symptoms at the time, but none at any subsequent period, although no attempt was ever made to restore the depressed bone to its natural situation, and I might add to the catalogue many. similar cases which have fallen under my own observation; but the fact is now well known to every practical surgeon; and in doing so, I should unnecessarily occupy the time and attention of the Society. Here the condition of the patient immediately after the accident does not indicate the necessity of an immediate operation; and a very interesting and important question arises as to the course which the surgeon should pursue, and whether he should, or should not, under these circumstances, resort to an operation for the purpose of elevating or removing the depression?

The removal of a part of the cranium is not to be viewed as a trifling matter, or as an operation which we are warranted in performing without a very sufficient reason. 1st. The process, by 
which the aperture in the cranium is filled up with new bone requires many years for its completion, even where the aperture is small; and where it is large, that process is never completed at all. The deficiency of the cranium must render the patient much more liable to suffer from accidental injury than he would have been if the cranium had been perfect. The cicatrix must be more easily penetrated by a cutting instrument, and more likely to give way under the force of a severe contusion than the bone itself; and in the second volume of the Edinburgh Medical Essays, a case is recorded in which, during a violent fit of the hooping-cough, such a cicatrix was lacerated, the dura mater torn, and the brain made to protrude through the wound, the patient dying with paralysis of the limbs five days afterwards. 2dly. Without referring to those remote consequences, or to cases in which it has been carelessly or improperly performed, the operation of the trephine is not to be regarded as one altogether free from danger. I saw a case in which a surgeon was induced to apply the trephine, although, as the event proved, there was no sufficient reason for so doing. The dura mater, at the time of the operation, was found adhering to the bone, and in a healthy state. Nevertheless, when the patient died some time afterwards, and the body was examined, the external layer of the circular portion of the dura mater which had been exposed in consequence of the trephine being employed, was seund in a state 
of slough, and it was a matter of doubt whether the sloughing did or did not extend through the whole thickness of the membrane. In another case, which occurred in St. George's Hospital, Mr. Gunning was induced to apply the trephine, in consequence of a suspicion that suppuration had taken place between the bone and the dura mater. The suspicion proved to be ill-founded : the dura mater was in a perfectly natural state, and there was bleeding from the sinall vessels on its surface after the renewal of the bone. The patient died afterwards in consequence of inflammation of the brain and pia mater. On dissection, besides the usual appearances produced by such inflammation, it was found that the circular portion of the dura mater which had been exposed in the operation was in a state of slough, the slough extending through its whole substance. Everywhere else the dura mater was in a natural state. It is reasonable to conclude that the sloughing of the dura mater in these cases was the consequence of it being deprived of its natural protection, and of the supply of blood which it receives through the vessels of the bone.

Now if the patient, whose case was mentioned last, had survived some time longer, what would have happened ? The slough of the dura mater would have separated, and the brain losing the support which it derives from this firm membrane, and having its vessels loaded with blood would, 
in all probability, have become protruded in the form of what is denominated a hernia cerebri. Such a protrusion would not indeed aggravate the danger of the case, where suppuration had already taken place within, but it might make the difference of life or death to the patient where the inflammation had not begun to terminate in this manner.

That the removal of a portion of the cranium may in itself be sufficient to make the patient liable to this formidable and dangerous disease of hernia cerebri, would appear sufficiently probable without any particular experience on the subject; and for evidence that this is actually the case, we need not go further than the Transactions of this Society. I allude to the very important paper by Mr. Stanley, published in the eighth volume of this work. In every one of four cases, which are here recorded, in which a portion of the bone of the cranium had been removed by the trephine or straight saw, the dura mater was found not to have suffered from the injury, yet a hernia cerebri presented itself some days afterwards. In one case it is distinctly stated that the dura mater was in a state of slough at the time of the protrusion beginning to take place; but it does not appear whether in the other cases it gave way in consequence of sloughing or ulceration. ..

Taking all these facts into consideration, we cannot refuse our assent to the proposition that 
the perforation of the skull, and the removal of a part of it is attended with a certain degree of anger, and the evidence hitherto adduced is in favour of the opinion, that $" s$ it is most prudent to abstain from the use of the trephine, where there is a fracture with depression of the cranium pro. ducing at the time no unfavourable symptoms."

But much may be said on the other side of the question; and at any rate there are other points to be considered before we can arrive at a positive conclusion on the subject.

1st. Although in some cases sloughing of the dura mater and hernia cerebri may follow the operation of the trephine, there are many other cases in which this never happens, the dura mater granulating, and the wound cicatrizing favourably.

2dly. Notwithstanding that a depression of the cranium is allowed to remain in many instances without it being productive of any bad consequences, there are numerous examples of such an injury being followed by extensive mischief. Suppuration takes place on the surface of the dura mater, an abscess is formed between that membrane and the bone, and ultimately (as I shall endeavour to explain on a future occasion), if the abscess has no opportunity of discharging itself externally, the inflammation extends to the parts below, and there is suppuration of the tunica arach- 
noides and pia mater, leading inevitably to the patient's destruction.

3dly. Where a depression of the cranium is allowed to remain, it sometimes happens that symptoms arise after a considerable lapse of time, which may even endanger the life of the patient, and which are to be attributed to the continuance of the depression, although it had occasioned no inconvenience in the first instance. I saw a well-marked and very instructive case of this kind several years ago under the care of Sir Everard Home, of which Sir Everard has published some account in the Philosophical Transactions for the year 1814. A gentleman received a blow on his head in consequence of having fallen from his horse, which occasioned a fracture and depression of one parietal bone. The depression was two inches and a quarter in its longest, and an inch and a half in its shortest diameter, and in one part nearly three quarters of an inch below the natural level: At the end of six weeks the early symptoms had subsided, and the patient was considered well. As soon however as he returned to his usual occupations, various nervous symptoms began to shew themselves which manifestly depended on the continued pressure on the brain. These symptoms instead of diminishing, increased in severity, and on some occasions were such as to occasion serious alarm; in consequence of which at the expiration of three years fiom the time of the accident Sir 
Everard was induced to remove nearly the whole of the depressed bone with the trephine. The wound cicatrized readily. The symptoms which existed before the operation were immediately relieved, and, as I have been informed, never res curred.

In this case the fracture and depression were very extensive, and probably these ultimate ill consequences, or secondary effects of the injury, may be avoided if we consider it as a general rule, that an extensive or deep depression should lead to the application of the trephine, although the same necessity does not exist where the depression is small.

This rule however affords us no assistance with respect to the greater danger arising from the chance of suppuration between the bone and the dura mater; this being as likely to occur where the depression is small as where it is large.

Sir Astley Cooper has stated in his Lectures on Surgery * that there is a great difference as to the danger of inflammation and suppuration of the membranes of the brain, between those cases in which the fracture and depression is complicated with a wound of the scalp, and those in which the

- The Lectures of Sir Astley Cooper, Bart. by F. Tyrrell, sic. Vol. 1. 
soft parts are uninjured; such mischief being much more liable to occur in cases of the first kind than in those of the second : and on these grounds he recommends that, where this complication exists, we should not hesitate to apply the trephine; and on the other hand, that, where it does not exist, we should carefully abstain from adding to the injury, by dividing the scalp and exposing the fracture. But many persons undoubtedly have recovered in whom there was at the same time a wound of the scalp, and a fracture and depression of the cranium, although no operation was resorted to. The cases to which $I$ have before alluded as published by Mr. Abernethy, are all examples of this fact ; and I recollect other similar cases which have fallen under my own observation. I have conversed also with several other surgeons whose experience on the subject has corresponded with my own, and all these circumstances led me in the first instance to doubt the accuracy of Sir Astley: Cooper's conclusion*.

* The following statement was furnished to me by my friend and colleague, Mr. Rose, from notes which he made whilesurgeon to the Coldstream Regiment of Guards during the Pe. ninsular war:-

"In the battle of Talavera de la Reyna, which was fought" on the 27th and 28th of July 1809, the brigade of guards lost about 600 men in killed and wounded. Amongst the latter were a considerable number of cases of wounds in the head. There were aigreat many cases of fracture of the bones of the craniam with and without depression, and from the cause which 
The question however is not to be decided merely on these premises. Many persons may do well without an operation who siffer from what Sir Astley Cooper denominates a compound fracture of the cranium, and yet it may remain to be determined what is the probability of suppuration taking place in these cases, as compared with those in which the scalp escapes uninjured?

For many years I have preserved notes of a large proportion of the cases of injury of the head, which

produced them, these were, of course, in every instance com. plicated with wounds of the scalp.

"On the $\cdot 3 d$ of August, in consequence of some military movements, the town of Talavera, in which the hospital had been formed, became exposed, and an order was given for all the wounded who could march, to leave it: This was so speedily obeyed that no time was afforded to make any selection. The worst cases necessarily remained, but among those who undertook the march there were twelve or fourteen with wounds in the head, accompanied with injuries of the bone, at least four or five of whom had both tables of the skull fractured, and two of them, along with fracture of the os frontis, had each the globe of one eye totally destroyed. In none of them had the trephine been applied, nor had any attempt been made to remove splinters of bone. After leaving Talavera, they were exposed to a burning sun, and to very severe fatigue. Every evening, after the day's march, Mr. Rose collected the wounded round him, examined and washed their wounds, dressing with care those that particularly required it. Cold water was the principal application employed. The retreat occupied sixteen days, in spite of which, and with no other treatment than that which has been described, every one of those who were wounded in the head recovered." 
it has fallen to my lot to witness. Among them, of course, are many in which there was fracture, with or without deprcasion, followed by suppuration between the dura mater and the bone. On referring to these for further evidence on this interesting subject, I find that the cases in which suppuration takes place where the scalp is entire have been comparatively rare; bearing a very small proportion indeed to those cases in which suppuration has followed a fracture complicated with a wound of the scalp. Such is the result of my own experience, during a considerable period of time, and which I am enabled to give not merely from a general recollection of what I have seen, but on the authority of written notes, made at the bedside of the patients, and for the most part before the question which they illustrate had ever presented itself to my mind.

Taking all these facts into consideration, and endeavouring to give its proper value to what may be urged on either side of the question, I cannot but acknowledge, whatever may have been my first impression on the subject, that it appears to me at this moment that the views of Sir Astley Cooper are well-founded; and that, in those cases in which a depression of bone exists without any symptoms, or with only trifling symptoms arieing from it, the surgeon can follow no better general rule than this: if the depression be exposed in consequence of a wound of the scalp, let him ap- 
ply the trephine, and elevate the depression : but if there is a depression without a wound of the scalp in consequence of the accident, let him not make such a wound by an operation. An exception may perhaps be properly made with respect to very extensive depressions of the cranium, which it may be prudent to expose and elevate at all events, not because there is a greater danger of suppuration from these than from smaller injuries, but on account of the ultimate ill consequences which the patient may experience if the brain be left permanently subjected to a very considerable pressure.

I have only two further observations to offer before $I$ leave this part of the inquiry.

The first is, that even where the fracture and depression of bone is complicated with a wound of the scalp, there is not, in all cases, the same absolute necessity for the application of the trephine. The bone may be depressed in such a manner as to allow the escape of the pus which is formed on the surface of the dura mater, although the depression is not elevated; or its position may be such as that the abscess can find no external opening. The danger in the first case must be infinitely greater than that in the second. A boy was admitted into St. George's Hospital who had received a severe blow on the head. The scalp was wounded, and there was a fracture and depression of bone: but as the depressed bone 
was not of a large size, as it was not much below its natural level, and as it produced no symptoms of importance, I did not apply the trephine. Eight or nine days after the accident, the boy complaining of pain in the head, the pulse having become frequent, and there being an expression of anxiety in the countenance, I divided the scalp beyond the wound which already existed, so as to expose the fracture more completely. I now discovered that suppuration had taken place beneath the bone, but the edge of the depressed bone was so much below the level of the bone in the neighbourhood that there was a very free opening for the escape of the pus, which was distinctly seen at the bottom of the wound rising and falling as the pulsations of the brain were communicated to it. No further operation was performed. The symptoms were relieved by the more free division of the scalp; the wound healed, and the patient left the hospital quite recovered about five weeks after his admission.

The last observation relates to a circumstance, the possible occurrence of which adds to my unwillingness to divide the scalp in cases of fracture and depression of the cranium, where it has not been already divided by the violence inflicted on it at the time of the accident. I have seen two cases in which the scalp remained entire, but in which the bone was fractured and depressed, and the dura mater lacerated, and the brain itself wounded by the edges of the fracture. Such a 
complication may be sufficiently dangerous under any treatment; but if we are to judge from the analogy of what occurs, not only in cases of simple and compound fractures of the extremities, but of other mechanical injuries, we must suppose that the danger would be much aggravated by the addition of a wound of the scalp. Suppuration of the brain and its membranes, to a greater or less extent, must necessarily ensue, if they are exposed under the circumstances which have been described, and it seems not improbable that such mischief may be avoided if the scalp be allowed to remain entire for their covering and protection. Of course this remark applies only to the conduct of the surgeon in the first instance. The treatment to be pursued, if, at a later period suppuration should be actually established, is not under our present consideration.

SEct. 9. Treatment of Contusions and Wounds of the Scalp.

As the treatment to be employed in cases of concussion and compression of the brain involves questions of peculiar interest, which demand the earliest attention of the surgeon in the greater number of instances of injury of the head, I have thought these subjects not undeserving of our. first consideration. It remains for us to determine the course which is to be pursued in other cases, of which the principal are; those of wounds and contusions of the scalp; of fracture, unattended with 
depreasion, and where there is no reason to believe that there is extravasation of blood beneath the bone; and those of wounds of the brain or its membranes.

Extravasation of blood in the cellular texture of the scalp seems to require for the most part no particular attention. Here, as elsewhere, the swelling made by the extravasation gradually becomes less prominent, and more diffused, and no great length of time elapses before it disappears altogether. I was consulted concerning the case of a young gentleman, in whom there was an effusion of blood under the scalp, extending from the superciliary ridges to the nape of the neck, and from ear to ear. When I saw the patient the blood appeared to be still in a fluid state, or at any rate not completely coagulated; and it had been poured out in such quantity that the cranium itself was not in any part perceptible to the touch : nevertheless, in the course of a few weeks, with no other application than that of a cold lotion, the whole tumor disappeared.

It is evident that, whatever was the vessel ruptured in this instance, it must have continued to bleed for a considerable time before so large an extravasation could have taken place. In another case in which a vessel under the scalp was bleeding in the same gradual manner, and threatening to produce similar results, I was 
enabled to ascertain the point at which the extravasation began, and by making pressure in this situation to stop its further progress. : The patient was a child who had received a blow on one temple, I believe, from the corner of a table. Soon afterwards the nurse observed a swelling in the part which had been struck, which however attracted but little attention at the time. On the following day the swelling had increased, and the parents brought the child to London, a journey of several miles. During the journey; the swelling became still larger, and when I was consulted soon after their arrival in London, it occupied the whole temple. I directed the child to be kept quiet, and the head to be bathed with a cold lo. tion. Next day, however, the swelling had extended orer a considerable part of the head adjoining the temple, presenting an appearance exactly similar to that which was observed in the ease last mentioned. I now inquired of the hurse, more particularly than I had done before, what was the exact spot at which the head had been struck, and in which the swelling was first discovered; and having ascertained this, I applied a' graduated compréss and bandage, such as is used after bleeding in the temporal artery: athd from this time there was no further increase of the swelling.

Punctured and incised wounds of the scalp require (in the first instatice at least) no spetu- 
liar treatment. - Nothing that has occurred in my own experience would lead me to believe that there is any reason why adhesive plaster should not be employed to approximate the edges of a wound of the scalp, as well as those of a wound elsewhere. Erysipelas not uncommonly follows a wound of the scalp, but it seems to me to occur equally, whether the wound is dressed with adhesive plaster or in any other manner.

When a portion of the scalp is separated in the manner of a flap, so as to expose the tendon of the occipito-firontalis muscle, or the pericranium, if it be carefully and neatly replaced, it will often become united by the first intention to the parts from which it has been separated. In many cases however there will be no adhesion, as where some time has elapsed before the wound has been dress. ed; or there has been considerable contusion; or the surface of the wound has been smeared with dirt; or other extraneous substance. In other cases there will be partial adhesions, some parts. of the wound becoming united while there is suppuration elsewhere; and (as I shall have occasion to observe hereafter) this state of things requires much attention on the part of the surgeon, lest the formation of abscesses in certain places should do injury to the pericranium and bone, and destroy the adhesions in the neighbourhood.

In those cases also, in which the pericranium is 
separated from the bone, it is for the most part right to replace the scalp, with the torn surfaces in contact, and to allow them to have the chance of becoming united, whatever that chance may be. Such union will not unfrequently take place even in the adult, where the bone is not exposed to a great extent, and the parts are nicely adjusted to each other; but there is much more reason to expect it in the young person, on account of the greater vascularity of the harder textures before the period of growth is concluded.

SEct. 10.-Treatment of Fractures of the Cranium unattended with Depression.

It seems to be the general opinion of modern surgeons that a fracture of the cranium, where there is no depression, and no evidence of any considerable extravasation between the dura mater and the bone, requires nothing beyond the strict antiphlogistic treatment, which ought to be resorted to in all cases of injury of the head. The fractured surfaces being here in contact are under circumstances the most favourable to the process of union, and the removal of a portion of the bone with the trephine must be regarded as a considerable, and as far as the fracture itself is concerned, $a$ wanton addition to the mischief already inflicted, which, instead of expediting, cannot fail materially to retard the patient's ultimate recovery. 
The application of the trephine, under these circumstances, has nevertheless been recommended by Mr. Pott; and I should be guilty of a serious omission if I were to pass over in silence a question of such importance, and relating to a point of practice which has received the sanction of such high surgical authority.

In the perusal of Mr. Pott's treatise on Injuries of the Head, we cannot but feel some degree of astonishment that that eminent surgeon should have resorted to an operation with so little hesitation in a number of cases, in which the existing symptoms were of trifling importance, and in which there was no evidence of immediate danger. It does not appear however that Mr. Pott, on these occasions, acted merely under the influence of his early prejudices, or of the example of those who had gone before him; and although not formally stated in his writings, the following argument may be deduced from them in favour of the practice which he recommended and adopted.

1st. The blow which occasions a fracture of the cranium, is likely to do such further injury to the vessels of the dura mater as may lead to inflam. mation and suppuration of the external surface of that membrane, and the formation of an abscess between it and the bone.

2dly. If such an abscess be formed without a 
free external opening, the case must terminate fatally.

3dly. If immediately after the accident a portion of bone be removed by the trephine, the pus formed afterwards on the surface of the dura mater is enabled to escape, and the danger arising from its confinement beneath the bone is avoided.

But it may be urged in opposition to this doctrine, 1st, That Mr. Pott seems, on the one hand, to have greatly over-estimated the danger of suppuration between the bone and the dura mater in cases of simple fissure of the cranium; and that such mischief will be avoided in the very great majority of cases, provided that, from the moment of the accident, the patient be kept in a state of perfect repose, on a spare diet, with the head cool, blood being taken occasionally from the arm, and these remedies being combined with the use of saline purgatives*.

' Whoever reads Mr. Pott's observations on this subject, and compares them with what is now seen in hospital practice, will, if $I$ am not mistaken, find good reason to believe that suppuration between the dura mater and the bone in consequence of a fracture, is less common at the present period than it was, when Mr. Pott wrote; a difference which may fairly be attributed to the more strict antiphlogistic treatment, which modern surgeons do not fail to adopt in all cases of injury of the head, whether the early symptoms be or be not of a dangerous description. 
2dly. That he seems on the other hand to have under-estimated the evils which may arise from the removal of a portion of the cranium, to which in fact no allusion is made in any part of his writings. On this subject it is needless to repeat the observations which $I$ have made in a former part of this paper.

sdly. That even if suppuration should take place between the bone and the dura mater, a watchful surgeon may generally detect the circumstance before pus has been formed to any great extent, and that the patient has still the chance of being preserved by the timely application of the trephine.

We can scarcely hesitate to admit that the reasons for abstaining from the use of the trephine under the circumstances which have been described are more conclusive than those which may be urged in favour of a more active treatment; it being at the same time borne in mind that cases of fracture of the cranium, even without depression, are always to be regarded with a jealous eye, especially where the scalp is wounded and the pericranium separated from the bone, it being in these cases especially that danger exists of the formation of matter between the dura mater and the bone. 
Sxcr. 11.-Treatment of Wounds of the Brain and its Membranes.

Although the condition of the patient who labours under a wound of the brain, or dura mater, is essentially different from that of one in whom no such wound exists, the general treatment required in these two orders of cases is nearly similar; and bleeding, purgatives, low diet, and a state of perfect repose form an important part of the remedies to be employed in cases of wounds, as well as in those of concussion and compression of the brain.

The object of the local treatment, where there is a wound of the brain or its membranes, is not so much to relieve the existing symptoms as to prevent future ill consequences, the principal of which are (as I shall shew hereafter), 1st, inflammation extending from the wound over the membranes of the brain, and producing an effusion of serum and pus; 2dly, inflammation, suppuration, sloughing, and dissolution of the substance of the brain ; 3dly, protrusion of the brain, in the form of what is commonly denominated a hernia cerebri.

A judicious surgeon will always bear in mind, that, especially on these occasions, the first rule of his art is not to add to the mischief already done. If splinters of bone have penetrated into 
the brain, and can be removed with perfect facility, and without the smallest additional disturbance to the injured organ, such removal cannot be improper, and may probably be useful. Many persons however have recovered, in whom an opposite practice has been pursued. I saw a gen. tleman in whom detached fragments of bone remained imbedded in the brain, many months after he had received a wound in the head from a pistol bullet, and who suffered scarcely at all from the injury. Do not such cases justify us in leaving splinters of bone untouched, where there is any kind of obstacle to their easy extraction? Are they not even sufficient to show that any other mode of proceeding would be improper, and that it is better to leave the patient to take his chance with the splinters lodged in the brain, than to commit the smallest additional violence in an endeavour to remove them ?

A similar observation may be made respecting depressions of bone when complicated with wound of the brain. If the edge of the depressed bone be imbedded in the substance of the brain, it may be proper to restore it to its natural level, provided that this can be readily accomplished with the forceps or elevator. But individuals have recovered, in whom a depression of bone has been allowed, under these circumstances, to remain without being elevated; and it cannot be advisable to risk this chance of recovery, whatever 
it may be, if the elevation requires the application of such a degree of force as is likely to cause the most trifling additional injury to the wounded brain. I have myself been led to doubt the expediency of applying the trephine in those cases in which there were no circumstances making the operation absolutely necessary. The motion of the saw must occasion more or less jar to the tender substance of the brain; and this, which may be of little consequence where the brain and its membranes are entire, may make a serious difference as to the degree of danger, where these parts are already lacerated and contused. There is, moreover, the same objection here as in other instances, to the removal of any considerable portion of the parietes of the cranium, namely, the liability which it occasions to the formation of a hernia cerebri.

The lodgement of a musket ball, or other foreign body in the substance of the brain, is undoubtedly a very serious occurrence, and one attended with the greatest danger to the patient. If the foreign body be of such figure and dimensions, and so situated, that while one extremity of it is inclosed within the cavity of the cranium, the other extremity projects externally, it may of course be extracted, and, probably, ought to be extracted at all risks. But with respect to a musket ball or pistol bullet lodged in the brain, it may be 
observed, first, that it rarely happens that it can be discovered and extracted even by the lightest and most practised hand, without such $\bullet$ degree of violence as must be in itself sufficient to produce a train of evils, which in all probability would terminate in death : and, secondly, that there are numerous instances of persons who have recovered, although the ball was allowed to remain in the brain; some of whom have suffered no more than they would have suffered from its being lodged in a less important part of the body. Taking all these things into consideration, ought we not to regard it as the general rule, that the extraction of a ball should not be attempted; an exception to the rule being made only in those cases, in which, from its more superficial situation and other circumstances, the extraction can be easily accomplished without the employment of force, and without adding in any degree to the mischief already done?

On the whole (according to the view which I am led to take of the subject), there seems to be in the very great majority of cases of wounded brain, more wisdom in resorting to negative, than to active local treatment. At any rate, as the restorative powers of the animal system are on all occasions the principal agents in the reparation of mechanical injuries, we cannot be wrong wherever there is a reason for doubt as to what 
should or should not be done, in leaving nature to take her own course, in trusting to her efforts rather than to human science and art.

My ow n experience, as far as it goes, is in favour of what is here recommended. I do not mean, however, to assert that what $I$ have seen of cases of wounded brain is in itself sufficient to justify me in forming these conclusions, unaided by a general knowledge of disease, and by arguments derived from analogy. In fact, the cases of wounds of the brain, which occur in the routine of a civil hospital, are so few in number compared with those of other injuries of the head ; they exhibit such numerous and various complications; and the proportion of recoveries from such wounds, whatever system is pursued, is so small (especially among adult patients), that it would be bold of any surgeon, engaged in the ordinary duties of his profession, to declare that he had been able to make a comparison of the different modes of treatment on such an extended scale, as would enable him to lay down rules of conduct founded wholly on his own practice and experience. The opportunities of military and naval surgeons must be, at certain periods, more considerable, but the circumstances under which they occur are very unfavourable to that minute observation and accurate judgement, which would be necessary to enable them to derive from their opportunities, all the advantages, which they might 
otherwise afford. Where the experience of individuals fails, we are called on to look for other sources of information. I have referred to all the cases of wounded brain recorded in the works quoted below *, and the general results which they exhibit will be found not uninteresting, if viewed in their relation to this point of surgical practice. These cases are thirty-eight in number, of which twenty-six terminated favourably, and twelve unfavourably. This, of course, affords no information as to the actual rate of mortality in cases of this description, the fatal cases being for the most part regarded as too much a matter of course to be worthy of publication; while a very different opinion is entertained respecting the cases of recovery. But the following facts afford some useful information as to the circumstances under which recovery takes place.

In nine cases of wounded brain in which the bone was fractured, but not depressed, no operation whatever was performed. In two of them the patients died; in the remaining seven they recovered.

- Mémoires de l'Académie Royale de Chirurgie.-Transactions of a Society for the Improvement of Medical and Chirurgical Knowledge.-Duncan's Medical Commentaries.-Duncan's Annals of Medicine.-Edinburgh Medical Journal.Medico-Chirurgical Transactions, Vol. I. to Vol. XII. inclusive. -Le Dran's Observations in Surgery.-Hennen's Military Surgery. - Collection d'Observations Cliniques par M. A: Petit. 
In fifteen cases no operation was performed, beyond that of removing some splinters of bone with the forceps. In five of these cases the patients died, while in ten the patients recovered.

In four cases the wound of the brain was complicated not only with fracture, but with depression of bone. In one of them in which the depressed bone was allowed to remain without being elevated, the patient recovered. In the three remaining cases the depression was elevated with the assistance of the trephine; and one of these patients recovered, and two of them died.

In ten cases a musket ball was lodged in the brain. In two of them the ball was extracted, and one patient recovered, while the other died. In the remaining eight cases the ball was allowed to remain, no attempt being made for its extraction, and two of these patients died, while six of them recovered. Of these last, however, one died several weeks afterwards of inflammation of the brain induced by intemperance in drinking, and another, after having been sufficiently well to resume his duties as a soldier, died in the course of the following year, of what was regarded as a coup de soleil.

It appears then that in fourteen out of twentysix patients who recovered, no operation whatever was resorted to, and that in ten of the remaining 
twelve, there was no operation beyond that of removing splinters of bone with the forceps. Of those in whom a ball was extracted from the brain one died, and one recovered; and of those in whom the ball was not extracted two died, and six recovered. It is needless to add, that the conclusions to be deduced from these statements illustrate and confirm the observations which have been already made as to the principles which should direct the surgeon in his treatment of these formidable injuries *.

There is one circumstance connected with this subject, which is too important to be passed over in silence, and which may very properly be mentioned in this place, as it must very materially influence us in the opinion which we give, at the time of the accident, as to the probability of the

- Since these calculations were made, a very interesting case has been published by Dr. Rogers in the thirteenth volume of the Medico-Chirurgical Transactions, in which the breech-pin of a gun was lodged in the anterior lobes of the cerebrum, and extracted at the end of twenty-eight days, the patient afterwards recovering. Here the foreign body remained in the substance of the brain until inflammation had been going on for an entire month, so that the brain and its membranes must have become extensively agglutinated and consolidated around it. The question as to the extraction of a foreign body after such a lapse of time, and under such circumstances, belongs more properly to another part of these inquiries; my observation at present being intended to be confined (as nearly as that can be done) to the treatment to be employed immediately or soon after the occurrence of the injury. 
patient's recovery. I have not been able to discover, among all the works which I have consulted, a single instance of recovery from a wound of the posterior lobes of the cerebrum, of the cerebellum, or medulla oblongata; and in the great majority of cases in which a cure has taken place the injury has been confined to the frontal bone, and that part of the brain which is covered and defended by it.

SECT. 12.-On the Treatment of some other Cases which are not included under the foregoing heads.

In those cases in which a particular class of sensations is destroyed or impaired, -as where deafness, or a loss of smell or taste follows an injury of the head,-I am not aware that advantage is to be expected from any particular mode of treatment, or that the use of any remedies is indicated beyond those which are resorted to in ordinary cases of concussion of the brain. The patient generally recovers the sensations of which the accident had deprived him in the course of one or two years; but his cure is to be attributed not so much to the skill of the surgeon, as to the restorative powers of his own system. All cases however do not prove equally fortunate in the result. I saw a gentleman in consultation with Dr. Francis Hawkins, who had lost his sense of smell in con. sequence of a blow on the head many years before, and in whom no improvement in this vol. XIY. 
respect bad. ever taken place. 、He was not enen sensible of the odour of valerian, although he could distinguish the medicine by the taste.

When a patient is affected with furious and raving delirium, blood should immediately be taken from the arm, and, if possible, in a full stream. I scarcely remember a single case, in which delirium of this kind, occurring soon after a blow on the head, did not yield to a copious blood-letting. The patient may very probably relapse into the same state, as soon as the first effects of the loss of blood have subsided, and it may be necessary to resort to the same means a second or third time, before the relief is permanent.

As there is reason to believe that convulsions and furious delirium occur under nearly parallel circumstances, we may expect that the treatment which is useful in cases of the former description, will also be useful in those of the latter. When convulsions occur soon after the accident, bloodletting is undoubtedly indicated. It is not however always easy, when the patient's limbs are thus moved and agitated, to succeed at once in the operation; and in many cases, the convulsions having been once established, they will continue for a certain period, notwithstanding that a considerable quantity of blood has been taken away, subsiding at last spontaneously. In a few instances (as has been already explained) con- 
vulsions occur at the expiration of some days from the time of the injury. At this later period they may exist in combination with inflammatory symptoms, which may require the further use of the lancet. But they may also exist independently of inflammation, being aggravated by any additional abstraction of blood, and subsiding on the patient being allowed to take some more substantial nourishment than that which had been allowed him previously. A case has been related in a former part of this paper, which will serve to illustrate this last observation. 